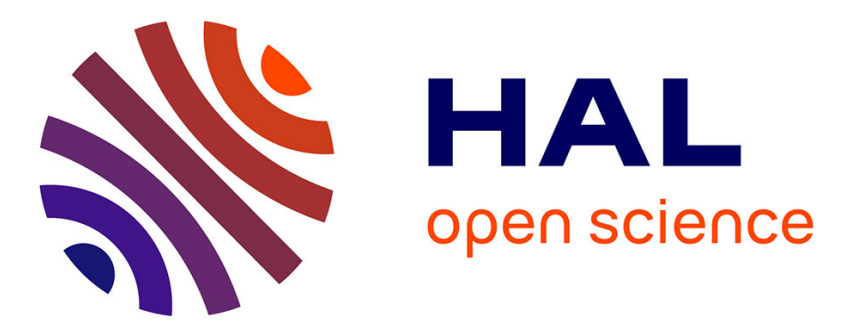

\title{
Human-Robot Team Interaction Through Wearable Haptics for Cooperative Manipulation
}

Selma Music, Gionata Salvietti, Pablo Budde Gen. Dohmann, Francesco Chinello, Domenico Prattichizzo, Sandra Hirche

\section{- To cite this version:}

Selma Music, Gionata Salvietti, Pablo Budde Gen. Dohmann, Francesco Chinello, Domenico Prattichizzo, et al.. Human-Robot Team Interaction Through Wearable Haptics for Cooperative Manipulation. IEEE Transactions on Haptics (ToH), 2019, 12 (3), pp.350-362. 10.1109/TOH.2019.2921565 . hal-02360685

\section{HAL Id: hal-02360685 \\ https://hal.science/hal-02360685}

Submitted on 13 Nov 2019

HAL is a multi-disciplinary open access archive for the deposit and dissemination of scientific research documents, whether they are published or not. The documents may come from teaching and research institutions in France or abroad, or from public or private research centers.
L'archive ouverte pluridisciplinaire HAL, est destinée au dépôt et à la diffusion de documents scientifiques de niveau recherche, publiés ou non, émanant des établissements d'enseignement et de recherche français ou étrangers, des laboratoires publics ou privés. 


\title{
Human-Robot Team Interaction Through Wearable Haptics for Cooperative Manipulation
}

\author{
Selma Musić Student Member, IEEE, Gionata Salvietti Member, IEEE, Pablo Budde gen. \\ Dohmann Student Member, IEEE, Francesco Chinello, Domenico Prattichizzo Fellow, IEEE, \\ Sandra Hirche Senior Member, IEEE
}

\begin{abstract}
The interaction of robot teams and single human in teleoperation scenarios is beneficial in cooperative tasks, for example the manipulation of heavy and large objects in remote or dangerous environments. The main control challenge of the interaction is its asymmetry, arising because robot teams have a relatively high number of controllable degrees of freedom compared to the human operator. Therefore, we propose a control scheme that establishes the interaction on spaces of reduced dimensionality taking into account the low number of human command and feedback signals imposed by haptic devices. We evaluate the suitability of wearable haptic fingertip devices for multi-contact teleoperation in a user study. The results show that the proposed control approach is appropriate for human-robot team interaction and that the wearable haptic fingertip devices provide suitable assistance in cooperative manipulation tasks.
\end{abstract}

\section{INTRODUCTION}

Reduction of price, size, and operational complexity of modern robots, as well as the advances in communication technology are enablers for multi-robot systems. Nowadays multi-robot systems are capable of performing many functions autonomously with high precision and repeatability. However, assistance by humans in high-level reasoning and (re-)planning is still needed. Human-robot team interaction is an interaction between single human and cooperative multi-robot system (robot team) [1]. It is particularly beneficial in teleoperation scenarios, where the robot team, executing a task in a remote environment, is controlled or supervised by the human.

One of the challenges in the human-robot team teleoperation is to resolve the interaction asymmetry, which occurs because the human operator typically has lower number of degrees of freedom than the robot team. Therefore, the robot team generally cannot be controlled by the human operator in its configuration space. Instead, it is necessary to establish the interaction on a higher level of abstraction. Another challenge is to provide force feedback signals to the human operator when the robot team interacts with environment through multiple contacts. Classical examples of such interaction are grasping and cooperative manipulation tasks. Kinesthetic, grounded haptic devices, typically used as interfaces in teleoperation,

S. Musić, P. Budde gen. Dohmann, and S. Hirche are with the Chair of Information-oriented Control, Technische Universität München, Munich, Germany, e-mail: \{selma.music, pablo.dohmann, hirche\}@tum.de

G. Salvietti and D. Prattichizzo are with the Department of Information Engineering and Mathematics, University of Siena, Italy, e-mail: salviettigio@dii.unisi.it, prattichizzo@ing.unisi.it

F. Chinello is with the Department of Business Development and Technology, Aarhus University, Denmark, e-mail: chinello@btech.au.dk cannot provide multi-contact feedback signals and have limited workspace. A solution to these problems could be to use cutaneous, wearable haptic devices, since they introduce a simplification of the hardware and mobility of the human.

In this article we analyze human-robot team interaction where a human operator, equipped with wearable haptic devices, teleoperates a robot team to perform a cooperative manipulation task. We propose a control scheme which establishes the interaction on spaces of reduced dimensionality. These spaces are determined through the modeling of robot team subtasks, necessary to accomplish a task, and represent a higher level of abstraction compared to the robot team configuration space. Subtasks may be conducted either sequentially or simultaneously. In order to ensure the human operator does not generate undesired motions nor receives undesired feedback signal through the haptic devices, decoupling of simultaneous subtasks is necessary and is achieved through the control design. We enhance the interaction by enabling the human operator to move freely in the workspace, which is achieved with wearable haptic fingertip devices. The control approach is validated experimentally. The influence of wearable haptic feedback on the task performance is evaluated in a user study.

This article extends the work published in [2] in the two following ways:

(i) A general decoupling control approach, suitable for heterogeneous robot teams.

(ii) A user study evaluation of different types of feedback mappings through wearable haptic devices in teleoperation for cooperative manipulation tasks.

Compared to the existing literature the contribution of this article is twofold. First, we propose a novel control approach for human-robot team interaction which resolves the problem of asymmetry by enabling the human operator to interact with the robot team on a higher level of abstraction through transformation-based mappings. This level is established through modeling and control of robot team subtasks on spaces of reduced dimensionality. Second, the suitability of wearable haptic devices in this type of interaction and for cooperative manipulation tasks is investigated for the first time.

The paper is organized as follows. Related literature is provided in Sec. II. The subtask modeling and the control scheme are proposed in Sec. III, while Sec. IV defines command and feedback mappings. The evaluation results are reported in Sec. V. Discussion of the results, obtained from the user study, is provided in Sec. VI, and conclusive remarks are given in Sec. VII. 


\section{RELATED WORK}

In this section we review the state-of-the-art related to human-robot team interaction for cooperative manipulation tasks. More specifically, we provide an overview of the existing control approaches for cooperative manipulation, task abstraction concepts that are relevant to our approach, and control architectures for teleoperation of robot teams. Works on existing command mappings and employed interaction interfaces in teleoperation of robot teams are also mentioned

Controller design for cooperative manipulation tasks includes two objectives: tracking desired trajectory of the object and zero internal loading on the object. The object dynamics is either assumed to be known [3] or approximated by an impedance [4], [5]. The problem of internal loading is solved through the control of internal forces which do not contribute to the object motion. The control approaches mainly differ in the way the internal forces are computed. For example, in [6] a virtual linkage physical model is proposed to describe internal forces, in [7] and [5] a pseudo-inverse of the grasp matrix is used, while the authors of [3] claim that an inertia-weighted pseudo-inverse of the grasp matrix is required. Majority of the approaches assume the object is part of the dynamical system. As a consequence, cooperative manipulation tasks further assume the object is already grasped (typically with a fixed grasp) and that the object dynamics is known. These assumptions are rather strong when operation in unknown environment is considered.

The concept of task abstraction has been introduced in robotics to reduce the dimensionality of the problem and enable development of high-level controllers. For example, in [8] a set of manipulation task primitives for single manipulators is introduced. Their combinations enable execution of different manipulation tasks. Similarly, task abstraction is employed on more complex robot systems (e.g. humanoids) through combinations of behavior primitives in [9]. Task abstraction concept is also applied to multi-robot systems by defining its global and local behaviors. For example, in [10] locked and shape behaviors are introduced for multiple robots to describe their team motion and their formation shape, respectively. In [11] similar task abstraction is used to control multi-robot system using null-space based behavioral control.

In human-robot interaction desired performance is achieved through a suitable combination of human capabilities and robot autonomy. Therefore, task abstraction also enables easier interfacing between the human operator and the robot system [12]. This is particularly beneficial when the teleoperated robot differs kinematically from the master manipulator/human. For example, in [13] a hidden robot concept is proposed in which the human operator performs a task in a virtual environment, translated into commands for the robot performing an actual task. Task abstraction in teleoperation is also achieved through virtual fixtures, see e.g. [14]. In the context of humanrobot team interaction and task abstraction, the human operator typically provides desired input for global behaviors, e.g. guides collective motion of the robot team, while the robots within the team autonomously maintain desired local behaviors, e.g. relative coordination [15]. This means that local interactions within the team are often uncontrollable to human inputs, i.e. the human operator is unable to assist or intervene in cases of unexpected events or failures of the autonomy [16]. For the teleoperation of robot teams, null-space based behavioral control is proposed in [17] with cooperative manipulation as a motivational example. However, interactions with environment are not considered. Teleoperation for a cooperative manipulation task is considered in [15] where a passivity-based control approach ensures desired performance of decoupled global and local behaviors. While global behavior is controlled by the human, local behavior is performed autonomously. However, a full-scale experiment is not conducted. A similar approach is employed for the teleoperation of a robot hand in [18] and [19].

Commands employed in human-robot team interaction are typically obtained through mappings, e.g. task-based [20] and synergy-based [21], or gestures, e.g. [22], [23], [24], and [25]. Feedback is often only visual. A lack of haptic feedback makes these solutions unsuitable when the robot team interacts with environment [26]. The approaches which use haptic interfaces resort to grounded, kinesthetic devices, e.g. master manipulators in teleoperation settings [15], [27], [28]. However, these devices have relatively low number of degrees of freedom compared to robot teams, causing interaction asymmetry. Furthermore, these devices cannot provide more than one interaction point which makes them unsuitable if the robot team interacts with environment through multiple contacts, e.g. in cooperative manipulation tasks. Additionally, they considerably limit the workspace of the human operator and can compromise stability in case of time delays and hard contacts. Glove-type displays such as the CyberGrasp system (CyberGlove Systems LLC, SanJose, CA, USA) can simultaneously reproduce contact forces in all the fingers, but the mechanics of these displays includes wires and bulky transmission systems that usually compromise the overall system wearability and portability.

Wearable displays represent a promising solution since they are able to apply cutaneous feedback to the fingerpads guaranteeing a high wearability and low encumbrance [29]. These devices have already been successfully used in a virtual telemanipulation setting where the human hand was tracked with a RGB-D camera and the slave side was represented by a virtual model of the human hand [30]. They have also been used to provide cues about shape geometry at the contact point [31]. The advantages of a wearable master system are twofold. Firstly, the master workspace is not limited by the workspace of the devices thanks to their extreme wearability and portability. This furthermore enables the simultaneous stimulation of several interaction points on the human hand. Secondly, the teleoperation system is intrinsically stable since finger tracking and force feedback are decoupled [32].

\section{CONTROL ARCHITECTURE FOR HUMAN-ROBOT TEAM INTERACTION}

In this section we first provide an overview of the humanrobot team system. Then, we propose a control architecture suitable for this type of interaction. 


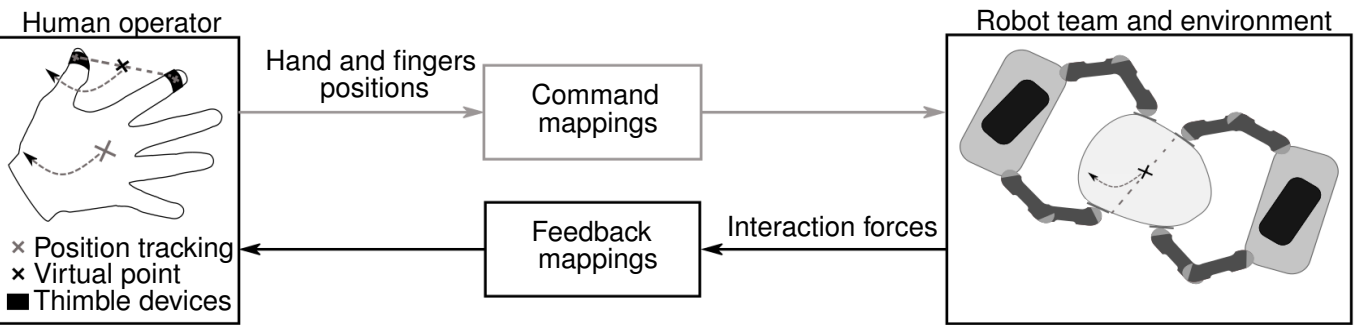

Fig. 1. The overall human-robot team interaction system. The human operator through hand and fingers motions and command mappings guides the robot team to grasp, manipulate and maintain the grasp of the object. Sensed interaction forces between the robots and the object are fed back through feedback mappings to the wearable haptic fingertip devices, mounted on the human operator fingers.

\section{A. Overview of the human-robot team system}

A teleoperation scenario where a human operator teleoperates a team of robot manipulators to grasp and manipulate an object is considered. Since the robot team can have a high number of controllable degrees of freedom compared to the human operator teleoperating it, a principal challenge is to resolve this interaction asymmetry through appropriate command mapping. If the robot team interacts with environment, e.g. an object, asymmetry may also arise if kinesthetic haptic devices are used since they may provide insufficient number of contact points to the human operator, making such interaction unobservable. In order to resolve this challenge, we propose a feedback mapping and the use of wearable haptic devices. The overall human-robot team interaction system for a cooperative manipulation task is depicted in Fig. 1.

Our approach is based on the assumption that the robot team performs the overall task through a set of subtasks. For example, in order to perform a cooperative manipulation task, the robot team needs to grasp, manipulate, and maintain the grasp of the object. The subtasks can be accomplished through modeling and controlling of the robot team subject to taskrelated constraints. In this context subtasks for cooperative manipulation can be achieved through cooperative and relative constraints [2]. Cooperative constraint ensures that the robot team is able to move as an entity (global behavior) and is necessary for the object manipulation subtask. Relative constraint ensures that the robot team has a specific formation shape (local behavior) which is necessary to maintain the grasp of the object. The concept of subtasks resolves the challenge of interaction asymmetry because it describes the system on a higher abstraction level than actuation level of the robot team. More precisely, the approach we propose enables modeling and control of the system on spaces of reduced dimensionality which simplifies human-in-the-loop control.

The proposed approach is not limited to the considered scenario. It may be applied to direct physical interaction as well, where the human interacts with the robot through a common, grasped object. Furthermore, the control approach proposed in this section and the mapping approach proposed in Sec. IV may be applied to any other robot team system, e.g. team of UAVs, wheeled robots, or a heterogeneous robot team. The task does not necessarily need to be cooperative manipulation, but may be any task that requires the team to maintain a specific formation shape while moving in its environment.

\section{B. Control of robot team subtasks}

In this subsection we define robot team subtasks and briefly propose control strategy suitable for cooperative manipulation. For a more detailed explanation of the control approach used the reader is referred to [2] and [33].

1) Robot team model: Let us assume that $N$ manipulators cooperatively grasp and manipulate an object, as shown in Fig. 2. The pose of the $i$-th manipulator is defined by the position and orientation of its end-effector in the Cartesian space, i.e. by the frame $\left\{c_{i}\right\}$ obtained w.r.t. the world frame $\{w\}$, described with the vector $\boldsymbol{x}_{i}=\left[\boldsymbol{p}_{i}^{\top}, \boldsymbol{q}_{i}^{\top}\right]^{\top}$, where $\boldsymbol{p}_{i} \in$ $\mathbb{R}^{3}$ is a position vector and $\boldsymbol{q}_{i} \in \operatorname{Spin}(3)$ is a unit quaternion.

The dynamics of the robot team is obtained by stacking $N$ Euler-Lagrangian equations

$$
\boldsymbol{M}(\boldsymbol{q}) \dot{\boldsymbol{v}}+\boldsymbol{c}(\boldsymbol{q}, \boldsymbol{v})+\boldsymbol{g}(\boldsymbol{q})=\boldsymbol{h}+\boldsymbol{h}^{m},
$$

where $\boldsymbol{M}(\boldsymbol{q})=\operatorname{blockdiag}\left(\boldsymbol{M}_{1}\left(\boldsymbol{q}_{1}\right), \ldots, \boldsymbol{M}_{N}\left(\boldsymbol{q}_{N}\right)\right)$ contains robots' inertial matrices, $\boldsymbol{c}=\left[\boldsymbol{c}_{1}^{\top}, \ldots, \boldsymbol{c}_{N}^{\top}\right]^{\top}$ stacks robots' Coriolis and centrifugal forces, and $\boldsymbol{g}=\left[\boldsymbol{g}_{1}^{\top}, \ldots, \boldsymbol{g}_{N}^{\top}\right]^{\top}$ stacks robots' gravitational forces. The control input and measured wrenches for each robot are stacked in $\boldsymbol{h}=\left[\boldsymbol{h}_{1}^{\top}, \ldots, \boldsymbol{h}_{N}^{\top}\right]^{\top}$ and $\boldsymbol{h}^{m}=\left[\boldsymbol{h}_{1}^{m \top}, \ldots, \boldsymbol{h}_{N}^{m \top}\right]^{\top}$, respectively. The measured wrenches are obtained through torque sensing of the robots and known Jacobian transformation from robots' joint space to Cartesian space. The stacked vectors of pose, velocity and acceleration are $\boldsymbol{x}, \boldsymbol{v}$, and $\dot{\boldsymbol{v}}$, respectively. The velocity vector of the $i^{\text {th }}$ robot is $\boldsymbol{v}_{i}=\left[\dot{\boldsymbol{p}}_{i}^{\top}, \boldsymbol{\omega}_{i}^{\top}\right]^{\top} \in \mathbb{R}^{6}$ with $\dot{\boldsymbol{p}}_{i}$ and $\boldsymbol{\omega}_{i}$ being translational and angular velocities of the endeffector, respectively. A wrench vector of the $i^{\text {th }}$ robot is $\boldsymbol{h}_{i}=\left[\boldsymbol{f}_{i}^{\top}, \boldsymbol{t}_{i}^{\top}\right]^{\top} \in \mathbb{R}^{6}$, with $\boldsymbol{f}_{i}$ and $\boldsymbol{t}_{i}$ being force and torque vectors, respectively. Therefore, the robot team has $n=6 \mathrm{~N}$ degrees of freedom in the Cartesian space. Through haptic devices typically up to 6 degrees of freedom can be controlled which is not enough in case the interaction is established with individual robots within the team. This is the reason why we reduce the dimensionality of the system by abstracting its behaviors. The joint angles of all the robots are stacked in the vector $\boldsymbol{q}$.

The main idea behind the approach is to define taskimposed, equality constraints, in the following form

$$
\boldsymbol{x}_{s, j}=\boldsymbol{f}_{j}(\boldsymbol{x}), \quad j=1, \ldots, r
$$

where $\boldsymbol{x}_{s, j} \in \mathbb{R}^{n_{j}}$ is the $j^{\text {th }}$ position and/or orientation constraint out of $r$ constraints with dimensionality $n_{j}$. Corresponding velocities are defined as

$$
\boldsymbol{v}_{s, j}=\boldsymbol{J}_{s, j}(\boldsymbol{x}) \boldsymbol{v}, \quad j=1, \ldots, r
$$




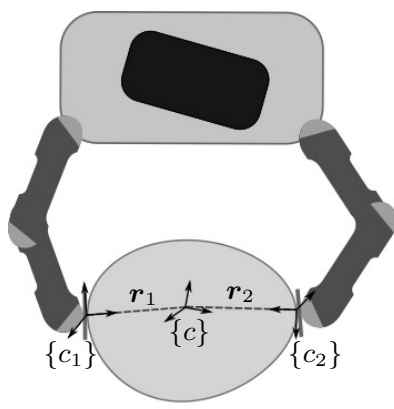

$\stackrel{f\{w\}}{\jmath}$

Fig. 2. Cooperative robot system grasps and manipulates a common object Frames $\left\{c_{i}\right\}, i=1,2$, are assigned to the robots' end-effectors and $\{c\}$ is the cooperative frame. Distances between $\left\{c_{i}\right\}$ and $\{c\}$ w.r.t. the world frame, $\{w\}$, are $\boldsymbol{r}_{i} i=1,2$.

where $\boldsymbol{J}_{s, j}(\boldsymbol{x}) \in \mathbb{R}^{n_{j} \times n}$ is the subtask Jacobian. The velocity of each robot can be computed through

$$
\boldsymbol{v}=\boldsymbol{P}_{s, 1}(\boldsymbol{x}) \boldsymbol{v}_{s, 1}+\ldots+\boldsymbol{P}_{s, r}(\boldsymbol{x}) \boldsymbol{v}_{s, r}
$$

where $\boldsymbol{P}_{s, j}(\boldsymbol{x}), j=1, \ldots, r$ are properly parametrized subtask Jacobian inverse matrices.

We use the subtask Jacobians as projection matrices to obtain maps of the robot team dynamics in lower dimensional spaces [33]. Let us now formulate relative and cooperative constraints for cooperative manipulation task conducted by two robots as depicted in Fig. 2.

2) Relative constraint: A suitable control of the robot team subject to the relative constraint allows the execution of the grasping and grasp maintenance subtasks. More specifically, it enables the robot team to establish a desired geometric formation shape in order to grasp and hold the object. Let us define the relative constraint as in (2) through the following translation and orientation equality constraints:

a) Translation constraints: Distances between the robots are explicitly defined in order for the robot team to obtain a formation shape

$$
\begin{aligned}
& \boldsymbol{p}_{r, 1}=\boldsymbol{R}_{1}{ }^{1} \boldsymbol{r}_{1,2}=\boldsymbol{p}_{1}-\boldsymbol{p}_{2} \\
& \vdots \\
& \boldsymbol{p}_{r, N-1}=\boldsymbol{R}_{N-1}{ }^{N-1} \boldsymbol{r}_{N-1, N}=\boldsymbol{p}_{N-1}-\boldsymbol{p}_{N},
\end{aligned}
$$

where $\boldsymbol{p}_{r, 1}, \ldots, \boldsymbol{p}_{r, N-1}$ are relative positions between the robots and $\boldsymbol{r}_{i, i+1}=\boldsymbol{r}_{i}-\boldsymbol{r}_{i+1}, i=1, \ldots, N-1$.

b) Orientation constraints: Relative orientation between the robots needs to be constrained to a constant value to avoid torsion and loss of contact

$$
\begin{aligned}
& { }^{2} \boldsymbol{q}_{1}=\boldsymbol{q}_{2}^{\star} \boldsymbol{q}_{1}=\text { const. } \\
& \vdots \\
& { }^{N} \boldsymbol{q}_{N-1}=\boldsymbol{q}_{N}^{\star} \boldsymbol{q}_{N-1}=\text { const. }
\end{aligned}
$$

Velocities computed from (5) and (6) to obtain expression as in (3) for $i=1,2$ as in Fig. 2 are

$$
\boldsymbol{v}_{r}=\underbrace{\left[\begin{array}{cccc}
\boldsymbol{I}_{3} & \boldsymbol{S}\left(\boldsymbol{r}_{1,2}\right) & -\boldsymbol{I}_{3} & \mathbf{0}_{3} \\
\mathbf{0}_{3} & \boldsymbol{I}_{3} & \mathbf{0}_{3} & -\boldsymbol{I}_{3}
\end{array}\right]}_{\boldsymbol{J}_{s, r}} \boldsymbol{v},
$$

where $\boldsymbol{v}_{r}=\left[\dot{\boldsymbol{p}}_{r, 1}^{\top}, \boldsymbol{\omega}_{r}^{\top}\right]^{\top}$ and $\boldsymbol{J}_{s, r}$ is the relative Jacobian.
3) Cooperative constraint: A suitable control of the robot team, subject to cooperative constraints, allows the execution of the object manipulation subtask. More precisely, it enables the robot team to move as a single entity and is defined with the frame $\{c\}$, depicted in Fig. 2, w.r.t. which all the robots within the team move cooperatively. Naturally, cooperative constraints can be satisfied only if the relative constraints are satisfied beforehand. Let us denote the pose of the frame $\{c\}$ w.r.t. $\{w\}$ with $\boldsymbol{x}_{c}=\left[\boldsymbol{p}_{c}^{\top}, \boldsymbol{q}_{c}^{\top}\right]^{\top}$. Let us further assume $\{c\}$ is located between the robots, with an arbitrary initial orientation. The cooperative constraint as in (2) is defined through the translation and orientation equality constraints.

a) Translation constraints: Based on the notations in Fig. 2 the following equality constraints on translations are imposed between the robots and the cooperative frame, $\{c\}$, in order for the robot team to move as an entity

$$
\boldsymbol{p}_{i}=\boldsymbol{p}_{c}+\boldsymbol{r}_{i}=\boldsymbol{p}_{c}+\boldsymbol{R}_{c}{ }^{c} \boldsymbol{r}_{i}, \quad i=1, \ldots, N
$$

where $\boldsymbol{R}_{c} \in \mathrm{SO}(3)$ is the rotational matrix of $\{c\}$ w.r.t. $\{w\}$.

b) Orientation constraints: In order for the robot team to rotate as an entity, relative orientation between the frames $\left\{c_{i}\right\}$, $i=1, \ldots, N$, and $\{c\}$ needs to be constant during manipulation

$$
{ }^{c} \boldsymbol{q}_{i}=\boldsymbol{q}_{c}^{\star} \boldsymbol{q}_{i}=\text { const. }, \quad i=1, \ldots, N
$$

where $\boldsymbol{q}_{c}^{\star}$ is a quaternion conjugate.

Computing velocities from (8) and (9) to obtain expression as in (4) if the relative constraints are satisfied, we obtain for $i=1,2$ as in Fig. 2

$$
\boldsymbol{v}=\underbrace{\left[\begin{array}{cc}
\boldsymbol{I}_{3} & \boldsymbol{S}^{\top}\left(\boldsymbol{r}_{1}\right) \\
\mathbf{0}_{3} & \boldsymbol{I}_{3} \\
\boldsymbol{I}_{3} & \boldsymbol{S}^{\top}\left(\boldsymbol{r}_{2}\right) \\
\mathbf{0}_{3} & \boldsymbol{I}_{3}
\end{array}\right]}_{\boldsymbol{P}_{s, c}} \boldsymbol{v}_{c},
$$

where $\boldsymbol{v}_{c}=\left[\dot{\boldsymbol{p}}_{c}^{\top}, \boldsymbol{\omega}_{c}^{\top}\right]^{\top} \in \mathbb{R}^{6}$ is the cooperative velocity vector, $\boldsymbol{S}($.$) is a skew-symmetric matrix, and \boldsymbol{P}_{s, c}$ is the projection matrix from the cooperative tangent space to the tangent space of the robot team.

Remark 1: The matrix $\boldsymbol{P}_{s, c}$ in (10) is equivalent to the grasp matrix if the actual object is tracked, as defined in [34].

Remark 2: If the robot team has $n=6 N$ degrees of freedom, according to (10) 6 degrees of freedom are needed to define cooperative subtask (through the pose of the frame $\{c\}$ ). In order to be able to achieve decoupling, relative behavior needs to be defined through $n-6$ degrees of freedom. We show that this is physically possible through the definition of relative constraints sequentially and not explicitly between all the robots yielding $n-6$ equations of relative constraints.

4) Subtask control: Our goal is to compute the contributions of the robot team dynamics to the subtasks defined through the cooperative and relative constraints. Evaluating robot team dynamics along the constraints reduces system dimensionality and simplifies control design. Furthermore, this enables discrimination between the interaction wrenches caused by different subtasks. With our control approach it is possible to obtain a separate representation of cooperative and 
relative subtask dynamics as in (1). For that purpose, we are able to obtain cooperative and relative velocity according to (4)

$$
\boldsymbol{v}=\underbrace{\left[\begin{array}{lll}
\boldsymbol{P}_{s, c}(\boldsymbol{q}) & \boldsymbol{P}_{s, r}(\boldsymbol{q})
\end{array}\right]}_{\boldsymbol{P}_{s}(\boldsymbol{q})} \underbrace{\left[\begin{array}{c}
\boldsymbol{v}_{c} \\
\boldsymbol{v}_{r}
\end{array}\right]}_{\boldsymbol{v}_{b}},
$$

where

$$
\boldsymbol{P}_{s, r}(\boldsymbol{q})=\boldsymbol{M}^{-1} \boldsymbol{J}_{s, r}^{\top}\left(\boldsymbol{J}_{s, r} \boldsymbol{M}^{-1} \boldsymbol{J}_{s, r}^{\top}\right)^{-1} .
$$

The acceleration is represented as

$$
\dot{\boldsymbol{v}}=\boldsymbol{P}_{s}(\boldsymbol{q}) \dot{\boldsymbol{v}}_{s}+\dot{\boldsymbol{P}}_{s}(\boldsymbol{q}) \boldsymbol{v}_{s} .
$$

Multiplying $\boldsymbol{P}_{s}^{\top}$ with (1) generates a decoupled representation of cooperative and relative subtask dynamics

$$
\begin{aligned}
& \boldsymbol{M}_{c}(\boldsymbol{q}) \dot{\boldsymbol{v}}_{c}+\boldsymbol{c}_{c}(\boldsymbol{q}, \dot{\boldsymbol{q}})+\boldsymbol{g}_{c}(\boldsymbol{q})=\boldsymbol{h}_{c}+\boldsymbol{h}_{c}^{m} \\
& \boldsymbol{M}_{r}(\boldsymbol{q}) \dot{\boldsymbol{v}}_{r}+\boldsymbol{c}_{r}(\boldsymbol{q}, \dot{\boldsymbol{q}})+\boldsymbol{g}_{r}(\boldsymbol{q})=\boldsymbol{h}_{r}+\boldsymbol{h}_{r}^{m},
\end{aligned}
$$

where $\boldsymbol{h}_{c}^{m}=\left[\boldsymbol{f}_{c}^{m \top}, \boldsymbol{t}_{c}^{m \top}\right]^{\top}$ and $\boldsymbol{h}_{r}^{m}=\left[\boldsymbol{f}_{r}^{m \top}, \boldsymbol{t}_{r}^{m \top}\right]^{\top}$ represent a contribution of the total wrenches to overcoming weight and inertia of the object and to the grasping maintenance, respectively.

In order to achieve desired performance of the defined subtasks, we propose to use position-based impedance controller which enables us to set desired error dynamics of cooperative and relative subtasks separately in the corresponding subspaces. Therefore, the desired cooperative and relative error dynamics are set as

$$
\begin{aligned}
& \boldsymbol{M}_{c}\left(\dot{\boldsymbol{v}}_{c}-\dot{\boldsymbol{v}}_{c}^{d}\right)+\boldsymbol{D}_{c}\left(\boldsymbol{v}_{c}^{d}-\boldsymbol{v}_{c}\right)+\boldsymbol{h}_{c}^{K}\left(\boldsymbol{x}_{c}^{d}, \boldsymbol{x}_{c}\right)=\boldsymbol{h}_{c}^{m} \\
& \boldsymbol{M}_{r} \dot{\boldsymbol{v}}_{r}+\boldsymbol{D}_{r}\left(\boldsymbol{v}_{r}^{d}-\boldsymbol{v}_{r}\right)+\boldsymbol{h}_{r}^{K}\left(\boldsymbol{x}_{r}^{d}, \boldsymbol{x}_{r}\right)=\boldsymbol{h}_{r}^{m},
\end{aligned}
$$

where $\boldsymbol{M}_{\boldsymbol{c}}$ and $\boldsymbol{M}_{\boldsymbol{r}}$ are the desired inertia matrices, $\boldsymbol{D}_{c}=$ blockdiag $\left[d_{c, t} \boldsymbol{I}_{3}, d_{c, r} \boldsymbol{I}_{3}\right]$ and $\boldsymbol{D}_{r}=\operatorname{blockdiag}\left[d_{r, t} \boldsymbol{I}_{3}, d_{r, r} \boldsymbol{I}_{3}\right]$ are the desired damping matrices, and the desired stiffnesses are given as in [5].

\section{MAPPING STRATEGY FOR HUMAN-IN-THE-LOOP INTERACTION WITH WEARABLE HAPTICS}

In this section a mapping strategy that enables the human operator to command desired motions to the robot team and to receive feedback from the robot team, interacting with the environment, through the wearable fingertip devices is proposed.

Following the approach introduced in [35] and further developed in [20], we define as command mapping a procedure necessary to provide desired control inputs to the robots within the team from the captured human operator motions. The feedback mapping is a procedure necessary to map interaction forces sensed on the robot team side to the haptic devices used at the master side. This approach establishes the human-robot team interaction on a higher level of abstraction, on which the human operator does not guide the robots individually but provides guidance to subtasks. Therefore, the approach does not depend on the number of robots within the team. In the remainder of the section we formulate the solution to the command and feedback mappings. For more details on the abstraction layer, readers can refer to [20].

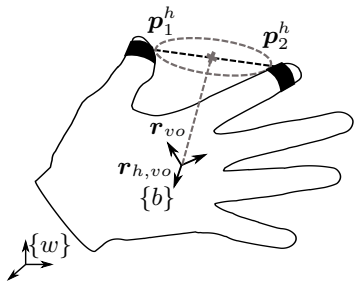

Fig. 3. The human hand and finger motions are mapped to inputs of the robot team subtasks. Frame $\{b\}$ is attached to the hand back. Positions of tracked fingers are $\boldsymbol{p}_{i}^{h}$ for $i=1, \ldots, M$. The distance vector between the frame $\{b\}$ and the virtual object is denoted as $\boldsymbol{r}_{h, v o}$, while the distance between the fingertips is $\boldsymbol{r}_{v o}$. Human fingertips are equipped with wearable haptic fingertip devices.

\section{A. Command mapping}

Let us assume the human operator is grasping a virtual object with $M$ contact points, $\boldsymbol{p}_{i}^{h} \in \mathbb{R}^{3}, i=1, \ldots, M$, as shown in Fig. 3. The virtual object is assumed to be a minimum volume sphere encircling all the contact points. Its pose is denoted as $\boldsymbol{x}_{v o}=\left[\boldsymbol{p}_{v o}^{\top}, \boldsymbol{q}_{v o}^{\top}\right]^{\top}$. Let us assume further that the pose of the human hand is known through the frame $\{b\}$ in $\{w\}$ and is denoted as $\boldsymbol{x}_{b}=\left[\boldsymbol{p}_{b}^{\top}, \boldsymbol{q}_{b}^{\top}\right]^{\top}$.

The pose of the virtual object can be computed from the hand pose

$$
\begin{aligned}
& \boldsymbol{p}_{v o}=\boldsymbol{p}_{b}+\boldsymbol{r}_{h, v o}=\boldsymbol{p}_{b}+\boldsymbol{R}_{b}{ }^{b} \boldsymbol{r}_{h, v o} \\
& { }^{b} \boldsymbol{q}_{v o}=\text { const. }
\end{aligned}
$$

From (16) the velocity of the virtual object, $\boldsymbol{v}_{v o}$, is

$$
\boldsymbol{v}_{v o}=\left[\begin{array}{cc}
\boldsymbol{I}_{3} & \boldsymbol{S}\left(\boldsymbol{r}_{h, v o}\right) \\
\mathbf{0}_{3} & \boldsymbol{I}_{3}
\end{array}\right] \boldsymbol{v}_{b},
$$

where $\boldsymbol{v}_{b} \in \mathbb{R}^{6}$ is the hand velocity. Desired cooperative velocity of the robot team is determined with the velocity of the virtual object

$$
\boldsymbol{v}_{c}^{d}=\boldsymbol{v}_{v o}
$$

The relative positions between the fingers are computed as in (5) with variables $\boldsymbol{p}_{r, 1}^{h}, \ldots, \boldsymbol{p}_{r, M-1}^{h}$ and their velocities $\dot{\boldsymbol{p}}_{r, 1}^{h}, \ldots, \dot{\boldsymbol{p}}_{r, M-1}^{h}$. We assume the virtual object can be moved and deformed through the change of contact points' positions. The consequence of this assumption is the variability of the virtual object radius

$$
\dot{r}_{v o}=\max \left\{\left\|\dot{\boldsymbol{p}}_{r, 1}^{h}\right\|, \ldots,\left\|\dot{\boldsymbol{p}}_{r,(M-1)}^{h}\right\|\right\} .
$$

Assuming that the directions of the robots' normals to the actual object at the slave side are known, the relative behavior of the robot team can be obtained through the change of the virtual object radius at the master side as

$$
\boldsymbol{v}_{r}^{d}=s_{r} \dot{r}_{v o} \boldsymbol{J}_{r, t} \hat{\boldsymbol{n}},
$$

where $s_{r}$ is the scaling factor which takes into consideration different workspace dimensions of the robot team and the human hand and $\boldsymbol{J}_{r, t}$ is the translation part of $\boldsymbol{J}_{r}$. The stacked vector of directions of normals in $\{w\}$ to the object surface for all robots is $\hat{\boldsymbol{n}}=\left[\hat{\boldsymbol{n}}_{1}^{\top}, \ldots, \hat{\boldsymbol{n}}_{N}^{\top}\right]^{\top}$. 


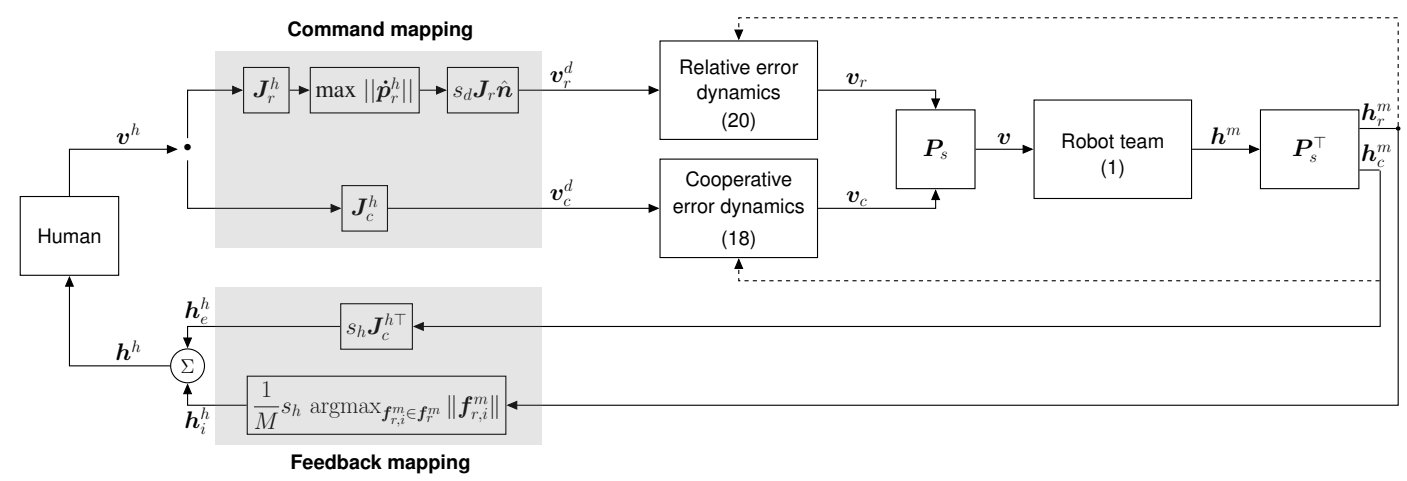

Fig. 4. Block structure of the control loop for human-robot team interaction.

\section{B. Feedback mapping}

Let us assume the human is equipped with $M$ wearable haptic fingertip devices as shown in Fig. 3. The feedback mapping is the procedure to compute the forces to feedback to each of the $M$ wearable devices. The computation of the forces to be feedback depends on the number of degrees of freedom of the used haptic devices and on the number of sensors available at the slave side. In this work, we use the wearable haptic thimble devices developed in [36] with 3 actuated degrees of freedom: roll, pitch, and displacement. We also consider that it is possible to compute the contact force of each robot. In the general case, there could be a mismatch between the forces measured at the slave side and those that can be displayed on the master side. For instance, it could be possible to measure wrenches for four arms on the slave side and only have two wearable devices on the master side that can only reproduce two forces. A possible solution for bilateral telemanipulation in case of dissimilar master and slave robots has been presented in [20].

The main idea of the proposed feedback mapping is to consider that the same wrench is applied both to the grasped object at the slave side and to the virtual object at the master side, considering a possible scaling factor $s_{h}=\frac{f_{\max }^{h}}{f_{\max }}$ determined by the ratio between the maximum wrench that can be rendered by the $M$ haptic devices $f_{\max }^{h}$, and the maximum wrench that is expected for the task $f_{\max }^{m}$. Therefore, the total wrench to be rendered at the master side can be computed as

$$
\boldsymbol{h}^{h}=s_{h} \boldsymbol{h}^{m},
$$

where $\boldsymbol{h}^{m}$ is the total measured wrench at the slave side. Also for the forces to be rendered at the master side it is possible to consider two contributions. One is related to the perception of the manipulated object weight and inertia and the other one is related to the feedback of the tightness of the grasp. As reported above, these two components are often referred to as external $\boldsymbol{h}_{e}^{h}$ and internal $\boldsymbol{h}_{i}^{h}$ forces. The external force contribution can be computed straightforward

$$
\boldsymbol{h}_{e}^{h}=\boldsymbol{J}_{c}^{h \top} \boldsymbol{h}_{c}^{m} .
$$

For the cooperative Jacobian on the master side following equality holds $\boldsymbol{J}_{c}^{h \top}=\left(\boldsymbol{P}_{c}^{h}\right)^{\dagger \top}$, where

$$
\boldsymbol{P}_{c}^{h \top}=\left[\begin{array}{ccccc}
\boldsymbol{I}_{3} & \mathbf{0}_{3} & \ldots & \boldsymbol{I}_{3} & \mathbf{0}_{3} \\
\boldsymbol{S}\left(\boldsymbol{r}_{1, v o}\right) & \boldsymbol{I}_{3} & \ldots & \boldsymbol{S}\left(\boldsymbol{r}_{M, v o}\right) & \boldsymbol{I}_{3}
\end{array}\right],
$$

where $\boldsymbol{r}_{i, v o}, i=1, \ldots, M$, is the vector between the contact point $i$ and the virtual object center point. The internal force contribution depends on the homogeneous part of the solution of eq. (21) that is not unique in general, see [20]. The solution of the homogeneous part is necessary to determine in which direction it is more convenient to render the forces related to grasp tightness. Among the possible solutions to compute internal force directions reported in [20], in this work the nullspace projector $\boldsymbol{P}_{s, r}^{\top}(\boldsymbol{x})$, defined as in (12) has been selected to determine the directions for internal forces. Once the directions are determined it is necessary to compute the magnitude of the rendered internal forces. We compute that amount as

$$
\boldsymbol{f}_{i}^{h}=\frac{1}{M} \underset{\boldsymbol{f}_{r, i}^{m} \in \boldsymbol{f}_{r}^{m}}{\operatorname{argmax}}\left\|\boldsymbol{f}_{r, i}^{m}\right\|,
$$

i.e., the relative force vector with the maximum norm is fed back to the human operator. Considering the proposed haptic thimbles it is only possible to render forces, thus only this contribution from $\boldsymbol{h}_{r}^{m}$ have been considered. A block diagram of the whole control loop for human-robot team telemanipulation is depicted in Fig. 4.

\section{EXPERIMENTAL EVALUATION}

In this section we present the developed experimental setup of the robot team teleoperation for cooperative manipulation task. The setup is used to conduct two experimental evaluations: 1) the performance of the proposed control architecture and 2) the suitability of wearable haptic fingertip devices for cooperative manipulation task. The evaluation of the haptic devices is performed through a user study, in which the effect of different types of feedback on various performance measures is studied.

\section{A. Experimental setup}

The experimental setup used for the evaluation is depicted in Fig. 5. The teleoperated robot system consists of two 7 DoF KUKA LWR 4+ manipulators, mounted on a common platform. The robot manipulators interact with a $1 \mathrm{~kg}$ boxshaped object. The interaction wrenches are estimated from the internal torque sensors and known robot Jacobian. On the joint level, local position controllers are used.

The human operator is equipped with two wearable haptic thimble devices, mounted on the thumb and index fingers of 


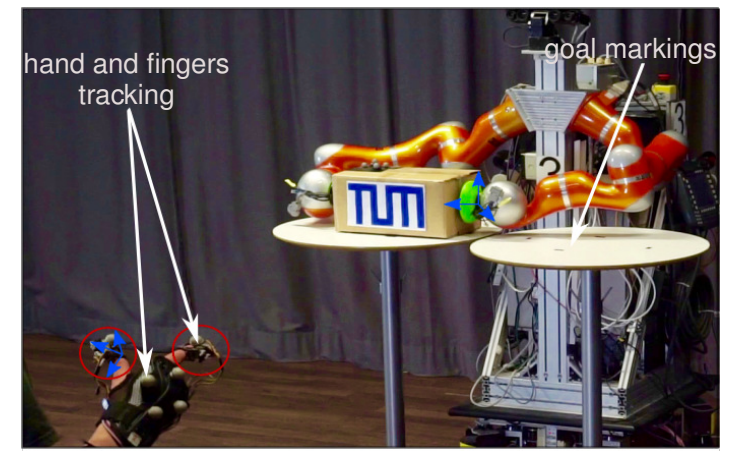

Fig. 5. Experimental setup: two KUKA LWR 4+ robot manipulators are teleoperated by a human operator, equipped with two wearable haptic thimble devices (marked with red circles). The coordinate frames of end-effectors and wearable fingertip devices are marke $\$$ Iin blue. THE CONTROL PARAMETERS.

\begin{tabular}{|l|c|c|}
\hline $\begin{array}{l}\text { Control } \\
\text { coefficients }\end{array}$ & $\begin{array}{c}\text { Cooperative } \\
\text { behavior }\end{array}$ & $\begin{array}{c}\text { Relative } \\
\text { behavior }\end{array}$ \\
\hline \hline$d_{c / r, t}$ & 200 & 300 \\
\hline$d_{c / r, r}$ & 5 & 5 \\
\hline$k_{c / r}$ & 500 & 500 \\
\hline$\kappa_{c / r}$ & 15 & 15 \\
\hline
\end{tabular}

the right hand. The operator guides the cooperative manipulation system to grasp and manipulate the object, according to the command mappings proposed in Section IV. For that purpose, the human operator is tracked with the marker-based motion tracking system, Qualisys. More specifically, 4 markers are mounted on the back of the right hand, and a marker is mounted on each thimble device. The number of markers is adequate for enabling the proposed interaction. No occlusions were present during the experiment execution.

Mechanical structure of the thimble device is depicted in Fig. 6 and its parts are denoted with capital letters. Three motors (E) control the length of the three tensors (B) which are connected to the vertices of the slanting platform $(G)$. The tensors independently pull the three vertices of the platform resulting in its 3 DoF: roll $(\alpha)$, pitch $(\beta)$, and displacement $(d)$. The device is equipped with FSR 400 force sensor $(\mathrm{H})$ which measures the contact force between the platform and the human finger. Operating principles of the thimble device are detailed in [32], [36], and [37]. The thimble devices are connected to a Raspberry Pi which ensures wireless communication with the robot system. The controller operates on $1 \mathrm{kHz}$, while the Qualisys motion tracking system and the fingertip devices operate on $0.1 \mathrm{kHz}$. Due to the absence of the kinesthetic feedback, the low sampling frequency of the wearable haptic thimble devices does not generate instability [32]. The control parameters are given in Table I. Feedback forces, $\boldsymbol{f}_{i}^{h}$ are mapped to the 3 degrees of freedom of the fingertip devices as

$$
\alpha=\operatorname{atan}\left(\frac{{ }^{t} f_{i, x}^{h}}{{ }^{t} f_{i, z}^{h}}\right), \beta=\operatorname{atan}\left(\frac{{ }^{t} f_{i, y}^{h}}{{ }^{t} f_{i, z}^{h}}\right), d=s_{h} k_{t} \sqrt{\left({ }^{t} \boldsymbol{f}_{i}^{h}\right)^{\top}{ }^{t} \boldsymbol{f}_{i}^{h}},
$$

with the scaling factor $s_{h}=\frac{4.7}{30}$ and the fingertip compliance parameter $k_{t}=2 \mathrm{~mm} / \mathrm{N}$ [38]. The poses of the coordinate frames $t_{j}, j=1, . ., M$, which are located at point $(\mathrm{H})$ in Fig. 6 are assumed to be known in $\{w\}$.
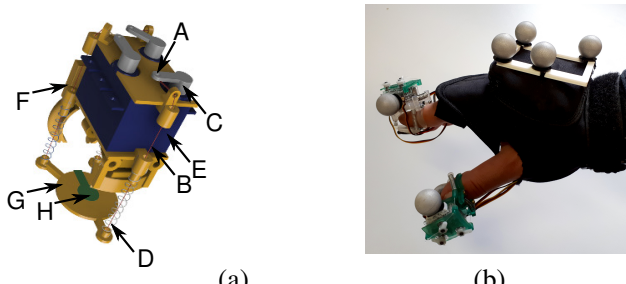

(a)

(b)

Fig. 6. The actuated fingertip device. (a) A rigid body (A) houses three servomotors (E) connected with three wires (B) to the vertices of a slanting surface $(G)$. The surface is located under the finger pulp of the user whose distal phalanx holds the fingertip in its position using a clamp (F). A force sensor $(\mathrm{H})$ is employed to set the calibration of the platform relatively to the finger size. The initial position of the platform is held by three springs (D) (b) Hand setup with the fingertip devices.
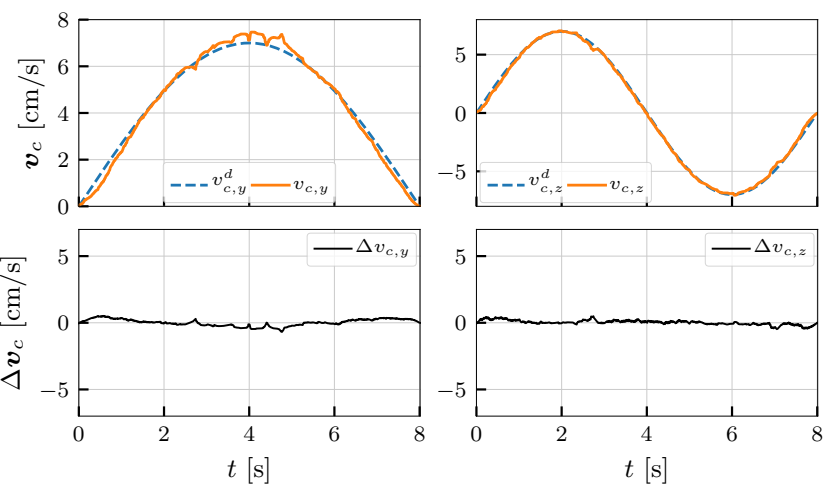

Fig. 7. Desired (- - ) and actual ( - ) cooperative velocities (top), $y$ (left) and $z$ (right), and their corresponding tracking errors (bottom), without human operator.

\section{B. Evaluation of the control architecture performance}

The proposed control approach is evaluated on an example of a pick and place task, that is conducted in the user study as well. For that purpose four experimental evaluations are performed:

(i) Tracking performance of cooperative behavior translation, without human in the loop, by applying $\boldsymbol{v}_{c}^{d}=$ $\boldsymbol{a} \sin (2 \pi \boldsymbol{f} t)$ with $\boldsymbol{a}=\left[\begin{array}{lll}0 & 0.07 & 0.07\end{array}\right]^{\top} \mathrm{m} / \mathrm{s}$ and $\boldsymbol{f}=$ $\left[\begin{array}{lll}0 & 1 / 16 & 1 / 8\end{array}\right]^{\top} \mathrm{Hz}$, resulting in a typical motion profile of a pick and place task in the $y-z$ plane of $\{w\}$.

(ii) Tracking performance of cooperative behavior rotation, without human in the loop, by applying $\omega_{c, z}^{d}=$ $a \sin (2 \pi f t)$, with $a=-0.07 \mathrm{rad} / \mathrm{s}$ and $f=1 / 6 \mathrm{~Hz}$.

(iii) Tracking performance of relative behavior, without human in the loop, by applying $v_{r, y}^{d}=0.05 \mathrm{~m} / \mathrm{s}$ during the approaching phase and $v_{r, y}^{d}=0 \mathrm{~m} / \mathrm{s}$ during the manipulation phase.

(iv) Tracking performance of a pick and place task, with human in the loop commanding the desired relative and cooperative trajectories and receiving relative force feedback through the haptic devices.

Experiment $(i)$ : The desired and actual cooperative velocity profiles in $y$ and $z$ directions, as well as the corresponding tracking velocity errors, are shown in Fig. 7. Good tracking can be observed with a mean error of $\left[\begin{array}{lll}\Delta \bar{v}_{c, y} & \Delta \bar{v}_{c, z}\end{array}\right]^{\top}=$ $\left[\begin{array}{ll}2.3 & 8.0\end{array}\right]^{\top} \times 10^{-5} \mathrm{~m} / \mathrm{s}$.

Experiment (ii): The desired and actual angular cooperative velocity profiles in $z$ direction, as well as the tracking velocity error, are shown in Fig. 8. Good tracking of angular velocity 

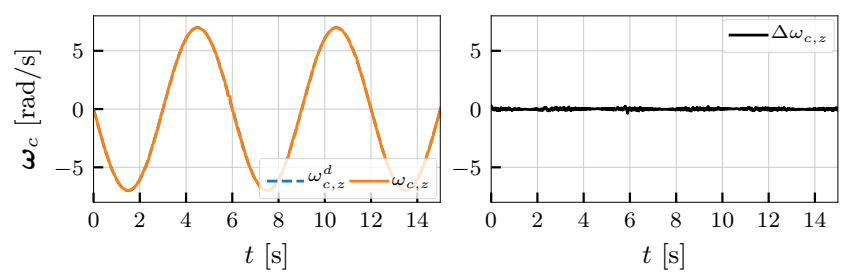

Fig. 8. Desired (- - -) and actual ( - ) cooperative angular velocities (left), $z$ component, and corresponding tracking error (right), without human operator.
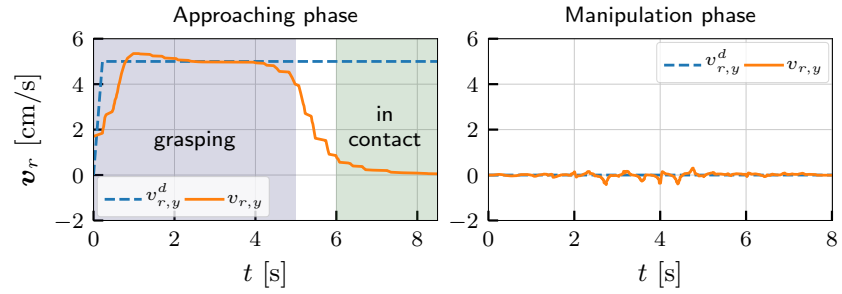

Fig. 9. Desired (- - $)$ and actual ( - ) relative velocities, $y$ component during approach to the object (left) and manipulation (right) phases, without human operator.

is achieved with a mean error of $\Delta \bar{\omega}_{c, z}=-1.8 \times 10^{-5} \mathrm{rad} / \mathrm{s}$.

Experiment (iii): The desired and actual relative velocity profiles during the approaching and the manipulation phases are shown in Fig. 9. After an initial overshoot producing an error of $\Delta v_{r, y}=3.2 \times 10^{-2} \mathrm{~m} / \mathrm{s}$, the desired velocity is achieved until the impact with the object. The transition from grasping (no contact) to grasp maintenance (contact) stages is smooth due to the proposed control strategy. The error between the desired and the actual velocities in contact is a consequence of the hard object constraints. Therefore, the actual velocity drops to zero as manipulators are no longer able to move relative to each other. As a result, potential energy is stored in the virtual spring of the impedance controller which maintains the grasp. After $t=8.5 \mathrm{~s}$ and in the "Manipulation phase" plot the desired relative velocity is set to $v_{r, y}^{d}=0 \mathrm{~m} / \mathrm{s}$. During the manipulation phase the mean relative velocity error is $\Delta \bar{v}_{r, y}=2.6 \times 10^{-3} \mathrm{~m} / \mathrm{s}$ confirming the noninteraction of the cooperative and relative behaviors.

Experiment (iv): The desired and actual cooperative velocity profiles, resulting from the motion commands of the human operator during a pick and place task are shown in Fig. 10. With the mean tracking velocity errors of $\left[\begin{array}{llll}\Delta \bar{v}_{c, x} & \Delta \bar{v}_{c, y} & \Delta \bar{v}_{c, z}\end{array}\right]^{\top}=\left[\begin{array}{lll}2.54 & 9.94 & 11\end{array}\right]^{\top} \times 10^{-4}$, it can be concluded that the desired velocities, provided by the human operator, are successfully tracked. Relative force profile, $f_{r, y}$, as well as the force in the local frame of the index fingertip device, ${ }^{t} f_{2, z}^{h}$, sensed by the human operator, are shown in Fig. 11. It can be observed that the profile of ${ }^{t} f_{2, z}^{h}$ follows

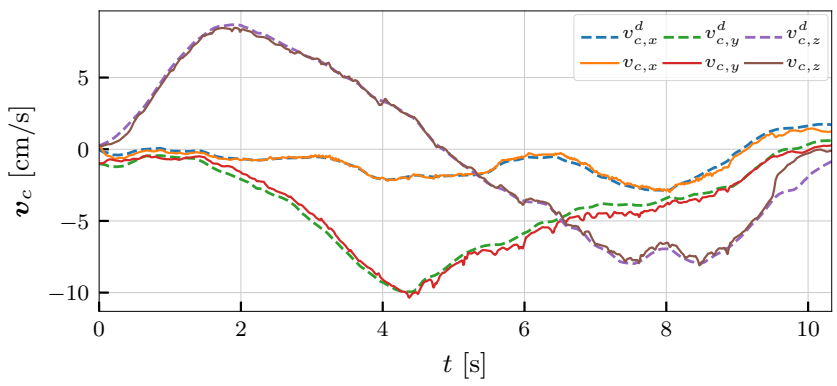

Fig. 10. Desired and actual velocities, $x, y$, and $z$ components, commanded by the human operator.

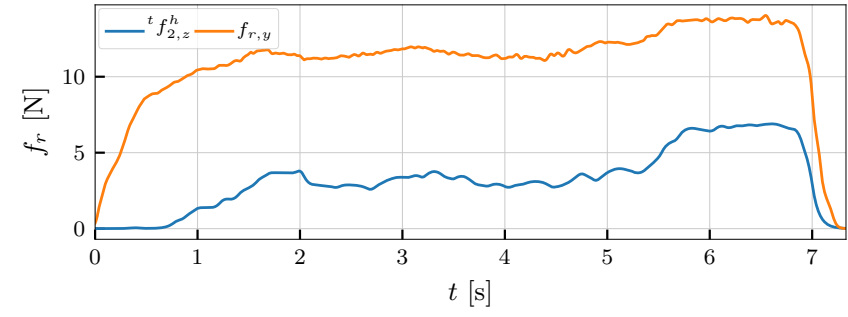

Fig. 11. Internal force measured by the robot team (- - -) along the $y$-axis and force sensed on the index thimble device platform (-). A stable grasp is maintained and reasonable transparency is achieved.

the profile of $f_{r, y}$, which indicates reasonable transparency of the system. However, possibly due to the curvature, the finger is not perfectly aligned with the force sensor. Therefore, low forces cannot be measured, resulting in the dead zone of ${ }^{t} f_{2, z}^{h}$ until $t=0.65 \mathrm{~s}$.

In this section we show that the performance of the proposed controller is satisfactory. Therefore, the human operator is able to command desired cooperative and relative velocities to accomplish grasp maintenance and object manipulation subtasks simultaneously. The decoupling of cooperative and relative subtasks is shown, as well as the applicability of the control approach in a realistic pick-and-place task. In the following subsection, we conduct a user study to evaluate the suitability of the wearable haptic fingertip devices in the presented experimental setup.

\section{User study design}

We conduct a user study to evaluate the performance of subjects in teleoperating a cooperative manipulation system using wearable thimble devices. We compare different types of feedback (independent variables) to understand which feedback type results in the best task performance.

Experimental task: The task is conducted on the experimental setup presented in V-A and shown in Fig. 5 with the control parameters reported in Table I. The participants were asked to perform the pick-and-place task in $y-z$ plane in $\{w\}$. In conducting the task, the users needed to command the grasping of the object to the robot manipulators, desired trajectory of the object while maintaining the grasp, and releasing of the object once the desired goal is reached. The initial and final poses are marked on the table. While conducting the task, the motion in $z$ direction, i.e. the height of the object during the motion, was not restricted to investigate how well the dexterity of the system is explored by the subjects. During the object manipulation phase, participants were not allowed to establish contact with the table or drop the object. Each participant performed the experiment ten times.

Independent variables: Four types of feedback mapped to the wearable thimble devices are tested:

1) No feedback $(N F)$ : Participants did not receive feedback through the fingertip devices. Instead, in order to conduct the task, they relied on the visual feedback only, i.e.

$$
\boldsymbol{f}_{i, 1}^{h}=\mathbf{0}_{3 \times 1} \text { and } \boldsymbol{f}_{i, 2}^{h}=\mathbf{0}_{3 \times 1}
$$

for the thimble devices mounted on the thumb and index fingers, respectively. 
2) Dynamic relative feedback (DF): Participants received feedback through the fingertip devices that corresponds to the scaled version of the measured relative forces. In particular, the measured relative force component is equally distributed to the $M$ thimble devices as defined by (22). The mapping of the feedback forces, $\boldsymbol{f}_{i}^{h}$, to the degrees of freedom of the thimble devices is achieved by (23).

3) Constant relative feedback $(C F)$ : Participants received a scaled constant feedback through the fingertip devices, determined as follows

$$
\boldsymbol{f}_{i, j}^{h}=\left\{\begin{array}{ll}
s_{h} 20 \mathrm{~N}, & \text { if }\left\|\boldsymbol{f}_{r}\right\| \geq 20 \mathrm{~N} \\
0 \mathrm{~N}, & \text { otherwise, }
\end{array} \quad j=1,2 .\right.
$$

The relative force of $20 \mathrm{~N}$ is sufficient to move the object. Therefore, this feedback type informed the human operator if it is safe to lift and move the object to the goal without slips and drops.

4) Binary feedback $(B F)$ : Participants received constant feedback through the fingertip devices, determined as follows

$$
\boldsymbol{f}_{i, j}^{h}=\left\{\begin{array}{ll}
s_{h} 7 \mathrm{~N}, & \text { if }\left\|\boldsymbol{f}_{r}\right\| \geq 7 \mathrm{~N} \\
0 \mathrm{~N}, & \text { otherwise, }
\end{array} \quad j=1,2 .\right.
$$

The relative force level of $7 \mathrm{~N}$ is not sufficient to move the object. Therefore, this type of feedback serves as a binary indicator for contact/no contact stages. As long as the contact is maintained, the feedback does not change. If the object drops, or is released, the feedback is $0 \mathrm{~N}$.

Subjects: Participants signed a written informed consent, approved by the ethics committee of the medical faculty of the Technical University of Munich. Forty eight healthy subjects (12 females and 36 males) participated in the user study. The participants were assigned to one of the four groups which correspond to the types of feedback. Twelve subjects were in each group.

Performance measures: The effect of different types of feedback is evaluated by the mean and maximum relative force measured by the robot system during the task execution, power-based effort, work, and mean absement of trajectory of the object in $z$ direction, i.e. total path traversed in $z$ direction over time.

1) Mean and maximum relative force: Mean relative force, measured by the robot manipulators during the interaction with the object, is computed as follows

$$
\bar{f}_{r, y}=\frac{\sum_{i=1}^{N} f_{r, y_{i}}}{N},
$$

where $N$ is the number of samples in the sequence. The maximum relative force applied is denoted as $f_{r, y}^{\max }=\max \left(f_{r, y}\right)$.

2) Power-based effort and work: The effort measure is calculated as power

$$
P=\overline{\dot{p}} \bar{f}^{m}
$$

where $\overline{\dot{p}}$ is the average translation velocity with $\dot{p}=\|\dot{\boldsymbol{p}}\|$, and $\bar{f}^{m}$ is the average measured force with $f^{m}=\left\|\boldsymbol{f}^{m}\right\|$.
The work done during the object manipulation stage is computed as follows

$$
W=\int_{0}^{T} P \mathrm{~d} t
$$

where $T$ is the duration of the object manipulation stage.

3) Absement: The area under the trajectory of the object motion in $z$ direction is computed with its absement

$$
A=\int_{0}^{T} x_{c, z} \mathrm{~d} t .
$$

Statistical tests and hypotheses: The mean across all subjects and all trials is computed for each performance measure to test the effects of different types of feedback. One-way analysis of variance (ANOVA) is performed and significant differences are accepted at $p<0.05$. A post-hoc test is carried out by Tukey-Kramer multiple comparison method. A posttest effect sizes are calculated to evaluate the practical significance of effects using Cohen's $d$ effect size measure using the calculation of effect sizes from ANOVAs with multiple groups in [39]. We test three hypotheses:

1) H1: Relative force feedback, i.e. DF and CF, improves task performance compared to no haptic feedback, NF.

2) H2: Relative force feedback, i.e. DF and CF, improves task performance compared to binary feedback, BF.

3) H3: Dynamic relative force feedback, DF, improves task performance compared to the constant relative force feedback, CF.

Data analysis: The recorded measurements were filtered with a second order Butterworth digital low-pass filter at a cut-off frequency of $6 \mathrm{~Hz}$. There were 13 error measurements. Multiple slips occurred and were detected from the drop in the profiles of recorded external force and its rate as in [40]. The slip force is detected from the recorded relative force at the point of the drop and is estimated to approximately $\left\|\boldsymbol{f}_{r}\right\|_{\text {slip }}=$ $9 \mathrm{~N}$. Measurements in which the system was uncontrollable by the subject were removed. Those are: measurements in which subjects were unable to control the relative subtasks because their thumb and index fingers were connected during the object manipulation, and measurements with accidental rotational slip since subjects were not able to control rotational degrees of freedom.

\section{Results of the user study}

Bar plots for all the performance measures are shown in Fig 12. The mean relative force measured by the robot system during the execution of the task is the lowest for dynamic and constant relative feedback types. The differences between the no feedback type and constant and dynamic feedback types are significant with large effect $\left(F_{3,344}=9.59, p<0.001, d=\right.$ 0.992), as depicted with the bar plot in Fig. 12(a). Post-hoc test revealed no significant differences between the dynamic and constant relative feedback types. The mean values of the relative force across 10 trials for all the conditions are shown in Fig. 13. It can be observed that the relative force levels are approximately constant for dynamic and constant feedback types across all trials and that they level out at approximately 


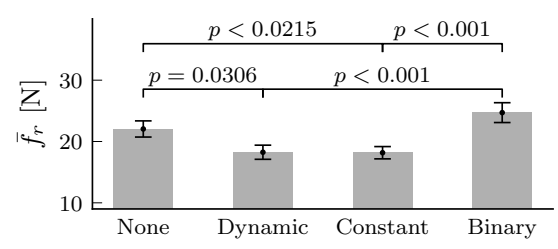

(a) Mean measured relative force.

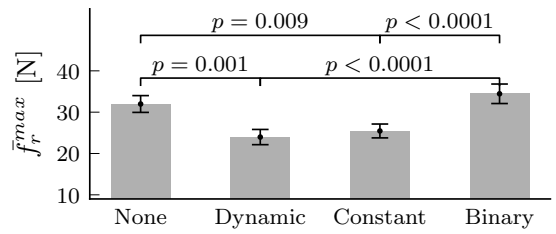

(b) Maximum measured relative force.

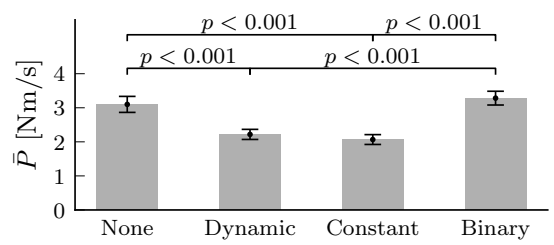

(c) Power-based effort.

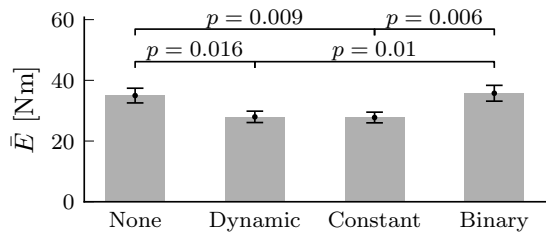

(d) Energy.

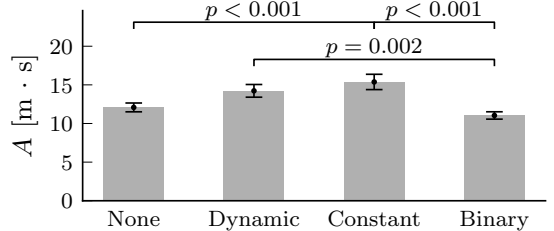

(e) Absement in meter-seconds.

Fig. 12. Bar plots showing the average values of relevant performance measures across 10 trials. The vertical bars in the plots are standard errors (SEs).

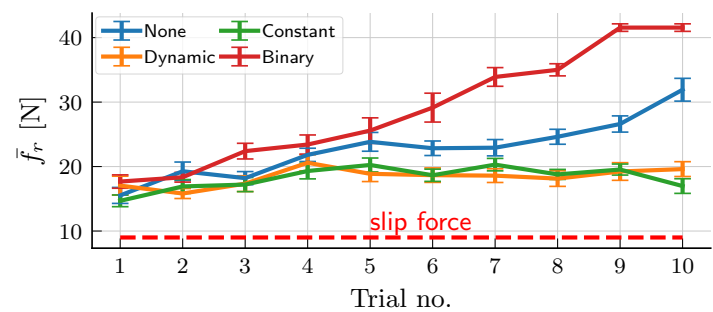

Fig. 13. Mean measured relative force performance measure across 10 trials.

$17.8 \mathrm{~N}$ and $18.6 \mathrm{~N}$, respectively. No feedback type has larger safety margin in comparison to the dynamic and constant feedback types and the relative force increases across trials. The profile of the relative force for the binary feedback type increases considerably with the number of trials which is not an optimal behavior during object manipulation. Maximum relative force, obtained during the lifting stage of the object, is the lowest for the dynamic and constant feedback types. These differences are significantly different with large effect $\left(F_{3,344}=10.29, p<0.001, d=0.995\right)$ from no feedback and binary feedback types, as denoted in Fig. 12(b).

The power-based effort and the total energy used to perform the task are the lowest for the dynamic and constant feedback types. ANOVA revealed significant difference for powerbased effort and the energy as well, $\left(F_{3,344}=17.59, p<\right.$ $0.001, d=1.225)$ with very large effect and $\left(F_{3,344}=\right.$ $6.47, p<0.001, d=0.736$ ) with medium effect, respectively. Significant differences obtained as a result of the multiple comparison post-hoc test are depicted in Fig. 12(c) and 12(d).

The absement of $x_{c, z}$ trajectory is the highest in the case of dynamic and constant feedback types, as shown in Fig. 12(e), i.e. that the participants traversed longer path while provided with either of these types of feedback. As a consequence they moved in a more dexterous manner and were able to use larger workspace of the robot team. This also means that, since there was no limit on the maximum height of the object, the participants were able to lift the object higher ANOVA showed a significant difference with medium effect $\left(F_{3,344}=10.0392, p<0.001, d=0.668\right)$.

\section{DISCUSSION}

H1. and H2. Relative force feedback improves task performance compared to no feedback and binary feedback.

Dynamic and constant relative feedback force types improve task performance compared to no feedback and binary feedback types. The mean relative force is significantly lower in the case of relative feedback types, as well as the maximum relative force during the lifting of the object. The effort representing the effect of the mean relative force and mean velocity of the manipulators is also significantly lower for the relative feedback types. The energy representing the effect of the effort and duration of the task is also the lowest for the relative force feedback types. Furthermore, with these feedback types, the subjects were able to lift the object the highest and traverse longer path. This means that the task was performed in a more dexterous manner, exploiting the robots' workspace better. Thus, $\mathrm{H} 1$ as well as $\mathrm{H} 2$ are confirmed.

H3. Dynamic relative feedback improves task performance compared to the constant relative force feedback.

No significant differences were observed between dynamic and constant relative feedback types in all the performance measures. Furthermore, performance for both feedback types was very similar. A possible reason may be that there was no interaction with the environment through the object. More specifically, if there were interactions (e.g. obstacles, holes, interactions due to occlusions, etc.), constant feedback type may not be able to convey the effect of the interaction force. On the other side, the dynamic feedback does not convey the information of the weight of the object and may be as good as the constant feedback for small object weights. The constant feedback is parametrized to be twice the amount of slip force, i.e. sufficient to manipulate the object. Therefore, it indirectly conveys the information of the weight of the object. The result also indicates the benefit of providing assistance through haptics, especially if the device is underactuated and cannot provide all task-relevant information. Namely, the constant feedback type informed the subjects that it is safe to lift and manipulate the object with the applied amount of force. This means that providing relevant assistance through haptics may significantly improve the task performance. Therefore, H3 is 
not confirmed but implies that assisting the human operator by providing relevant information through haptics provides satisfactory results.

\section{CONCLUSION}

In this article we propose a control architecture for humanrobot team interaction in cooperative manipulation tasks. The control approach is based on the concept of subtasks, which represent task-based constraints imposed on the system. We show that grasping, grasp maintenance, and object manipulation subtasks can be conducted along cooperative and relative constraints. Through the decoupling of the subtasks, we show that it is possible to compute grasping nd object-dynamics wrenches in a dynamically consistent way. Furthermore, through the appropriate command and feedback mappings, the control approach enables interaction between a human operator and the system on a higher level of abstraction. Wearable fingertip devices are used as haptic interfaces to enable teleoperation in a multi-contact setting. We evaluate user performance with different types of feedback and show that dynamic feedback of grasping forces improves task performance compared to no haptic feedback.

In this work we preliminarily focus on the teleoperation scenario for cooperative manipulation task. We plan to extend the approach to multiple subtasks and explore more complex application scenarios, e.g. transition from teleoperation to direct physical interaction, regrasp, and recovery in the case of failures of individual robots. Based on the findings of this work and previous literature, we believe it is good to divide a task so that the complementary capabilities of the human and the robots are exploited. By complementary capabilities we mean planning and robustness of humans in unknown environments and high precision of robots in repetitive tasks. Therefore, we believe that planning towards the goal, replanning in case of changes in the environment, reaction to events, and similar subtasks, should include human in the loop. The subtasks which require high precision, either in motion or force applied, and are a priori known during the task execution should be conducted by the robots. Other future directions may be towards a semi-autonomous control architecture, multimodal interfaces, manipulation of more complex and flexible objects, and a detailed evaluation of the subtask-based control approach.

\section{ACKNOWLEDGMENT}

The work is partly funded from DFG within the Joint Sino-German research project "COVEMAS", the DFG priority program SPP1914 "Cyber-Physical Networking", and H2020 European project "SoftPro: Synergy-based Open-source Foundations and Technologies for Prosthetics and Rehabilitation, Grant Agreement no. 688857.

\section{REFERENCES}

[1] S. Musić and S. Hirche, "Control sharing in human-robot team interac tion," Annual Reviews in Control, vol. 44, pp. 342-354, 2017.

[2] S. Musić, G. Salvietti, P. Budde gen. Dohmann, F. Chinello, D. Prattichizzo, and S. Hirche, "Robot team teleoperation for cooperative manipulation using wearable haptics," in Intelligent Robots and Systems (IROS), IEEE/RSJ International Conference on, 2017, pp. 2556-2563.
[3] S. Erhart and S. Hirche, "Model and analysis of the interaction dynamics in cooperative manipulation tasks," IEEE Transactions on Robotics, 2016.

[4] S. Schneider and R. Cannon, "Object impedance control for cooperative manipulation: Theory and experimental results," IEEE Transactions on Robotics and Automation, 1992.

[5] F. Caccavale, P. Chiacchio, A. Marino, and L. Villani, "Six-dof impedance control of dual-arm cooperative manipulators," IEEE/ASME Transactions on Mechatronics, vol. 13, no. 5, pp. 576-586, Oct 2008.

[6] D. Williams and O. Khatib, "The virtual linkage: A model for internal forces in multi-grasp manipulation," in Robotics and Automation, 1993 IEEE International Conference on, 1993, pp. 1025-1030.

[7] R. G. Bonitz and T. Hsia, "Internal force-based impedance control for cooperating manipulators," IEEE Transactions on Robotics and Automation, 1996

[8] J. D. Morrow and P. K. Khosla, "Manipulation task primitives for composing robot skills," in Proceedings of International Conference on Robotics and Automation, vol. 4, April 1997, pp. 3354-3359 vol.4.

[9] L. Sentis and O. Khatib, "Synthesis of whole-body behaviors through hierarchical control of behavioral primitives," International Journal of Humanoid Robotics, vol. 2, no. 04, pp. 505-518, 2005.

[10] C. Belta and V. Kumar, "Abstraction and control for groups of robots," IEEE Transactions on Robotics, vol. 20, no. 5, pp. 865-875, Oct 2004

[11] G. Antonelli, F. Arrichiello, and S. Chiaverini, "The null-space-based behavioral control for autonomous robotic systems," Intelligent Service Robotics, vol. 1, no. 1, pp. 27-39, 2008

[12] C. Belta, A. Bicchi, M. Egerstedt, E. Frazzoli, E. Klavins, and G. J. Pappas, "Symbolic planning and control of robot motion [grand challenges of robotics]," IEEE Robotics Automation Magazine, vol. 14, no. 1, pp. 61-70, March 2007

[13] A. Kheddar, C. Tzafestas, and P. Coiffet, "The hidden robot concept-high level abstraction teleoperation," in Proceedings of the 1997 IEEE/RSJ International Conference on Intelligent Robot and Systems. Innovative Robotics for Real-World Applications., vol. 3, 1997, pp. 1818-1825.

[14] D. Aarno, S. Ekvall, and D. Kragic, "Adaptive virtual fixtures for machine-assisted teleoperation tasks," in Proceedings of the IEEE International Conference on Robotics and Automation, 2005, pp. 1139-1144.

[15] D. Lee and M. W. Spong, "Bilateral teleoperation of multiple cooperative robots over delayed communication networks: theory," in Proceedings of the 2005 IEEE International Conference on Robotics and Automation. IEEE, 2005, pp. 360-365.

[16] D. Sieber and S. Hirche, "Human-guided multirobot cooperative manipulation," IEEE Transactions on Control Systems Technology, pp. 1-18, 2018.

[17] T. Hatanaka, N. Chopra, J. Yamauchi, and M. Fujita, "A passivity-based approach to human-swarm collaboration and passivity analysis of human operators," in Trends in Control and Decision-Making for Human-Robot Collaboration Systems. Springer, 2017, pp. 325-355.

[18] P. Michelman and P. Allen, "Shared autonomy in a robot hand teleoperation system," in Proceedings of IEEE/RSJ International Conference on Intelligent Robots and Systems, vol. 1, Sep. 1994, pp. 253-259 vol.1.

[19] W. Griffin, W. Provancher, and M. Cutkosky, "Feedback strategies for telemanipulation with shared control of object handling forces," Presence, vol. 14, no. 6, pp. 720-731, 2005

[20] G. Salvietti, L. Meli, G. Gioioso, M. Malvezzi, and D. Prattichizzo, "Multicontact bilateral telemanipulation with kinematic asymmetries," IEEE/ASME Transactions on Mechatronics, vol. 22, no. 1, pp. 445-456, Feb 2017.

[21] G. Gioioso, G. Salvietti, M. Malvezzi, and D. Prattichizzo, "Mapping synergies from human to robotic hands with dissimilar kinematics: an approach in the object domain," IEEE Transactions on Robotics, vol. 29, no. 4, pp. 825-837, 2013.

[22] C. Hu, M. Q. Meng, P. X. Liu, and X. Wang, "Visual gesture recognition for human-machine interface of robot teleoperation," in Proceedings 2003 IEEE/RSJ International Conference on Intelligent Robots and Systems, vol. 2, Oct 2003, pp. 1560-1565 vol.2.

[23] G. Gioioso, A. Franchi, G. Salvietti, S. Scheggi, and D. Prattichizzo, "The flying hand: a formation of uavs for cooperative tele-manipulation," in IEEE International Conference on Robotics and Automation, 2014.

[24] M. T. Wolf, C. Assad, M. T. Vernacchia, J. Fromm, and H. L. Jethani, "Gesture-based robot control with variable autonomy from the jpl biosleeve," in IEEE International Conference on Robotics and Automation, 2013, pp. 1160-1165.

[25] J. Nagi, A. Giusti, F. Nagi, L. M. Gambardella, and G. A. Di Caro, "Online feature extraction for the incremental learning of gestures in human-swarm interaction," in Robotics and Automation (ICRA), 2014 IEEE International Conference on. IEEE, 2014, pp. 3331-3338. 
[26] H. I. Son, A. Franchi, L. L. Chuang, J. Kim, H. H. Bulthoff, and P. R. Giordano, "Human-centered design and evaluation of haptic cueing for teleoperation of multiple mobile robots," IEEE Transactions on Cybernetics, vol. 43, no. 2, pp. 597-609, April 2013.

[27] Y.-C. Liu, "Task-space coordination control of bilateral human-swarm systems," Journal of the Franklin Institute, vol. 352, no. 1, pp. 311-331, 2015.

[28] E. J. Rodríguez-Seda, J. J. Troy, C. A. Erignac, P. Murray, D. M. Stipanovic, and M. W. Spong, "Bilateral teleoperation of multiple mobile agents: Coordinated motion and collision avoidance," IEEE Transactions on Control Systems Technology, vol. 18, no. 4, pp. 984-992, 2010

[29] C. Pacchierotti, S. Sinclair, M. Solazzi, A. Frisoli, V. Hayward, and D. Prattichizzo, "Wearable haptic systems for the fingertip and the hand: Taxonomy, review, and perspectives," IEEE Transactions on Haptics, vol. 10 , no. 4, pp. 580-600, Oct 2017.

[30] S. Scheggi, L. Meli, C. Pacchierotti, and D. Prattichizzo, "Touch the virtual reality: Using the leap motion controller for hand tracking and wearable tactile devices for immersive haptic rendering," in $A C M$ SIGGRAPH 2015 Posters. ACM, 2015.

[31] A. Frisoli, M. Solazzi, F. Salsedo, and M. Bergamasco, "A fingertip haptic display for improving curvature discrimination," Presence: Tele operators and Virtual Environments, vol. 17, no. 6, pp. 550-561, 2008.

[32] C. Pacchierotti, L. Meli, F. Chinello, M. Malvezzi, and D. Prattichizzo, "Cutaneous haptic feedback to ensure the stability of robotic teleoperation systems," International Journal of Robotics Research, vol. 34 no. 14, pp. 1773-1787, 2015.

[33] S. Musić and S. Hirche, "Passive noninteracting control for human-robot team interaction," in 2018 IEEE 57th Annual Conference on Decision and Control $(C D C)$, Dec 2018.

[34] D. Prattichizzo and J. C. Trinkle, Grasping. Berlin, Heidelberg: Springer Berlin Heidelberg, 2008, pp. 671-700.

[35] G. Salvietti, L. Meli, G. Gioioso, M. Malvezzi, and D. Prattichizzo, "Object-based bilateral telemanipulation between dissimilar kinematic structures," in IEEE/RSJ International Conference on Intelligent Robots and Systems (IROS), 2013

[36] D. Prattichizzo, F. Chinello, C. Pacchierotti, and M. Malvezzi, "Towards wearability in fingertip haptics: A 3-DoF wearable device for cutaneous force feedback," IEEE Transactions on Haptics, vol. 6, no. 4, pp. 506516, Oct 2013

[37] F. Chinello, M. Malvezzi, C. Pacchierotti, and D. Prattichizzo, "Design and development of a 3RRS wearable fingertip cutaneous device," in 2015 IEEE International Conference on Advanced Intelligent Mechatronics (AIM), July 2015, pp. 293-298

[38] K.-H. Park, B.-H. Kim, and S. Hirai, "Development of a soft-fingertip and its modeling based on force distribution," in IEEE International Conference on Robotics and Automation, vol. 3, 2003, pp. 3169-3174.

[39] W. Lenhard and A. Lenhard, "Calculation of effect sizes," Dettelbach (Germany): Psychometrica, 2016. [Online]. Available: https://www.psychometrica.de/effect_size.html

[40] Y. Hiramatsu, D. Kimura, K. Kadota, T. Ito, and H. Kinoshita, "Control of precision grip force in lifting and holding of low-mass objects," PLOS ONE, vol. 10, no. 9, pp. 1-19, 092015 .

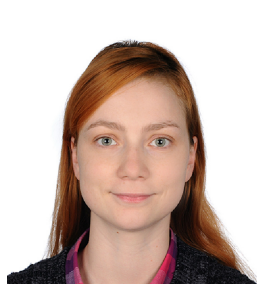

Selma Musić received her M.Sc. degree in Electrical engineering from the University of Sarajevo, Bosnia and Herzegovina in 2014. Since 2015 she is working towards a Doctor of Engineering degree in Electrical and Computer Engineering at the Chair of Information-oriented Control, Department of Electrical and Computer Engineering, Technical University of Munich (TUM), Germany. Her research interests include human-robot interaction, haptics, and control engineering.

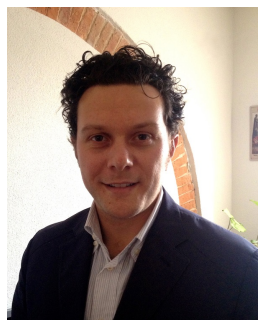

Gionata Salvietti (M12) received the M.S. degree in Robotics and Automation and the Ph.D. degree in Information Engineering from the University of Siena, Siena, Italy, in 2009 and 2012, respectively. He was a post-doc researcher with the Istituto Italiano di Tecnologia from 2012 to 2015 . He is currently Assistant Professor at Department of Information Engineering and Mathematics, University of Siena and Research Affiliate at Dept. of Advanced Robotics at Istituto Italiano di Tecnologia. From 2016, Associate Editor IEEE Robotics and Automation Letters. His research interests are telemanipulation, robotic and human grasping, haptics and assistive devices.

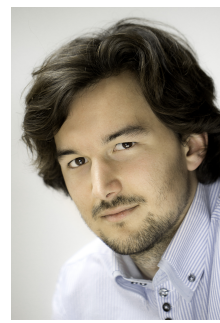

Pablo B. gen. Dohmann Pablo B. gen. Dohmann received his M.Sc. in Electrical and Computer Engineering from the Technical University of Munich, Germany in 2018. Since then he is pursuing the Dr.Ing. degree in Electrical and Computer Engineering at the Chair of Information-Oriented Control, Department of Electrical and Computer Engineering, Technical University of Munich. His research interest include multi-robot systems, networked and distributed control.

Francesco Chinello received the M.S. degree in Computer Engineering (focus on Robotics and Industrial automation) in 2010 from the University of Siena with a thesis entitled System software for rapid prototyping of anthropomorphic manipulators. On February 2014, he received his Ph.D. on Information Engineering. After that, he has been PostDoc at the SIRSLab (University of Siena) and guest researcher at the Department of Advanced Robotics (Italian Institute of Technology). Since January 2017, he is an Assistant Professor of the Department of Business Development and Technology, Aarhus School of Business and Social Science, at Herning (Denmark).

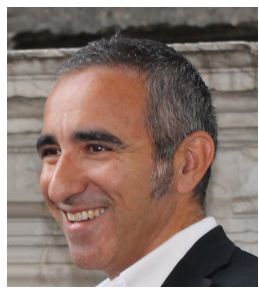

Domenico Prattichizzo received the Ph.D. degree in Robotics and Automation from the University of Pisa in 1995. Since 2015 Full Professor at the University of Siena. From 2002 to 2015 Associate Professor of Robotics at the University of Siena. Since 2009 Scientific Consultant at Istituto Italiano di Tecnologia, Genova Italy. He is a fellow of the IEEE and he is President of Eurohaptics Society. In 1994, he was a Visiting Scientist at the MIT AI Lab. Since 2014, he is Associate Editor of Frontiers on Robotics and AI. From 2007 to 2013 he has been Associate Editor in Chief of the IEEE Transactions on Haptics. From 2003 to 2007, he has been Associate Editor of the IEEE Transactions on Robotics and IEEE Transactions on Control Systems Technologies. He has been Chair of the Italian Chapter of the IEEE RAS (2006-2010), awarded with the IEEE 2009 Chapter of the Year Award. Research interests are in haptics, grasping, mobile robotics and geometric control. He has been the Coordinator of the IP collaborative project WEARable HAPtics for Humans and Robots (WEARHAP).

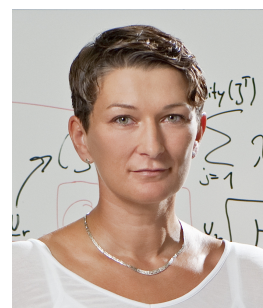

Sandra Hirche (M'03-SM'11) received the DiplomIngenieur degree in aeronautical engineering from Technical University Berlin, Germany, in 2002 and the Doktor-Ingenieur degree in electrical engineering from Technical University Munich, Germany, in 2005. From 2005 to 2007 she was awarded a Postdoc scholarship from the Japanese Society for the Promotion of Science at the Fujita Laboratory, Tokyo Institute of Technology, Tokyo, Japan. From 2008 to 2012 she has been an associate professor at Technical University Munich. Since 2013 she is TUM Liesel Beckmann Distinguished Professor and heads the Chair of Information-oriented Control in the Department of Electrical and Computer Engineering at Technical University Munich. Her main research interests include cooperative, distributed and networked control with applications in human-robot interaction, haptics, multi-robot systems, and general robotics. She has published more than 150 papers in international journals, books and refereed conferences. Dr. Hirche has served on the Editorial Boards of the IEEE Transactions on Control of Network Systems, IEEE Transactions on Control Systems Technology, and the IEEE Transactions on Haptics. She has received multiple awards such as the Rohde \& Schwarz Award for her PhD thesis in 2005, the IFAC World Congress Best Poster Award in 2005, Best Paper Awards at IEEE Worldhaptics and the ERC Starting Grant "Control based on Human Models" (2014-19). 